\title{
CADENA Marisol de la, Earth beings: ecologies of practice
} across Andean worlds

Duke University Press, Durham/London, 2015, xxvII-340 p., bibliogr., ilustraciones en blanco y negro, mapa.

\section{Pablo F. Sendón}

\section{OpenEdition}

Journals

Edición electrónica

URL: https://journals.openedition.org/jsa/14726

DOI: $10.4000 /$ jsa. 14726

ISSN: 1957-7842

Editor

Société des américanistes

Edición impresa

Fecha de publicación: 31 octubre 2016

Paginación: 235-243

ISSN: 0037-9174

Referencia electrónica

Pablo F. Sendón, «CADEnA Marisol de la, Earth beings: ecologies of practice across Andean worlds», Journal de la Société des américanistes [En línea], 102-1 | 2016, Publicado el 05 diciembre 2016, consultado el 02 septiembre 2022. URL: http://journals.openedition.org/jsa/14726 ; DOl: https:// doi.org/10.4000/jsa. 14726

Este documento fue generado automáticamente el 2 septiembre 2022.

All rights reserved 


\section{CADENA Marisol de la, Earth beings: ecologies of practice across Andean worlds}

Duke University Press, Durham/London, 2015, XXVII-340 p., bibliogr., ilustraciones en blanco y negro, mapa.

\section{Pablo F. Sendón}

\section{REFERENCIA}

CADENA Marisol de la, Earth beings: ecologies of practice across Andean worlds, Duke University Press, Durham/London, 2015, xxvII-340 p., bibliogr., ilustraciones en blanco y negro, mapa.

Este libro recoge para el ámbito andino una problemática novedosa - aunque, desde una perspectiva más general, quizás la novedad no resida tanto en la problemática en sí como en la manera en que se la ha abordado - acerca de la cual la antropología dedicada a otras latitudes - entre la que destaca una parte de la que se ocupa de las así llamadas «tierras bajas » sudamericanas - se ha venido pronunciando de manera insistente durante los últimos años: las diferentes expresiones ontológicas de la relación hombre-naturaleza ${ }^{1}$. En términos de la casuística abordada - resultado de conversaciones y experiencias compartidas entre la autora y sus « amigos » (p. Xv passim) Nazario Turpo y su padre -, a diferencia del estado-nación en el que están insertas y que regula buena parte de sus vidas, entre las poblaciones campesino-indígenas y quechua hablantes que habitan el macizo del Ausangate en el sur del Perú no existe hiato, solución de continuidad, salto o quiebre entre el universo de los seres humanos (o runakuna) y el de los «seres-tierra » (« earth-beings» o tirakuna, p. xxiII passim), debido a que estas poblaciones «son-enayllu ( (« being-in-ayllu», p. 44 passim), siendo, el ayllu, « el colectivo de runakuna y tirakuna » (p. 12). 
2 La « ontología histórica del conocimiento moderno » (p. 13) resulta una dificultad para adentrarse en concepciones del mundo y formas de actuar en él que le son ajenas. Entre los campesinos quechua hablantes del Ausangate la ausencia de quiebre o de ruptura no sólo entre los hombres y los « seres-tierra ", sino también entre la narración y el evento, la palabra y la cosa, el nombre y el ser, el significante y el significado, etc., provoca un sinnúmero de equívocos que no son ajenos a la labor de traducción en antropología. Sin embargo, el « equívoco » (" equivocation», p. 27) resulta « una dimensión constitutiva del proyecto disciplinar de traducción cultural » (ibid.) y, a partir de él es posible alcanzar «conexiones parciales» (" partial connections», p. 31) que permiten hacer explícitas, sin anularlas, diferencias ontológicas entre sociedades y culturas diferentes, o entre sectores culturales diferentes de una misma sociedad. El libro no sólo se detiene en el reconocimiento y profundización, descripción mediante, de estas diferencias; también identifica y se pronuncia acerca del desconocimiento, negación o indiferencia de la sociedad mayor ante ellas y las consecuencias, políticas inclusive, que todo esto trae aparejado.

3 El libro consta de dos secciones cuyos capítulos (rebautizados «historias») relatan eventos sobresalientes en las vidas de Mariano Turpo y su hijo - hoy fallecidos - en sus respectivos roles de, en el primer caso, líder indígena de un movimiento enfrentado a una de las más importantes haciendas de la región antes de la reforma agraria de 1969 y, en el segundo, chaman andino receptivo a las demandas de un movimiento no menos importante como el turismo místico contemporáneo a gran escala y curador de la sección quechua del National Museum of the American Indian en Washington, cubriendo así un espectro histórico que, grosso modo, comienza en la década de 1920 y culmina en la actualidad.

4 Mariano Turpo nació en la aldea de Pacchanta (distrito de Ocongate, provincia de Quispicanchi ${ }^{2}$, Cuzco) que, durante las primeras tres cuartas décadas del siglo pasado, era un ayllu de una de las parcialidades o sectores que conformaban la hacienda Lauramarca con la que estuvieron en pleito prácticamente desde su constitución. Además de yachaq ${ }^{3}$, Mariano fue el personero responsable de servir a su ayllu en el conflicto que lo enfrentó con la hacienda durante el proceso de reforma agraria y en los movimientos que la precedieron. He aquí un primer episodio en la historia de los equívocos y conexiones parciales en las que se concentra el libro - así como también de lo que se pierde en el proceso de traducción: los « seres-tierra » y todo aquello que les está asociado. Si bien la tierra era uno de los principales objetos del conflicto, ella « no sólo era el terreno agrícola del que los campesinos se ganaban la vida; también era el lugar donde estaban los tirakuna y los runakuna» (p.110); he aquí un equívoco. Ahora bien, «en tanto convergencia de ambos, la tierra fue el término que permitió la alianza entre mundos radicalmente diferentes y parcialmente conectados » (ibid.), el mundo de la política y el mundo del ayllu; he aquí una conexión parcial. El territorio por el que peleaban estos mundos parcialmente conectados no era lo mismo o, en otros términos, la palabra que servía para designarlo se refería a cosas diferentes. La reforma agraria, y el movimiento que la precedió, no sólo fue un conflicto político; también fue un " conflicto ontoepistémico» (p.111) en el que los logros alcanzados por el ayllu permanecieron desconocidos, «en las sombras en donde los esfuerzos del ayllu hicieron posible el evento histórico » (ibid.); he aquí una conclusión.

5 Mariano conservaba en su hogar una caja con unos cuantos centenares de documentos de diversa índole, la mayoría consistentes en denuncias a las transgresiones de las reglas de 
convivencia llevadas a cabo por parte de representantes regionales y locales del Estado durante las décadas en el que se desarrolló el conflicto con la hacienda. La autora caracteriza a estos documentos como " archivo » y propone discutir su «intrincación ontológica » (« ontological intricacy », p. 121) ya que ellos se descubren en la «frontera onto-epistémica » (« onto-epistemic border », p. 122) entre el Estado y el ayllu. La idea es la siguiente: el archivo "fue el lugar donde la historia y las prácticas ahistóricas ["ahistorical worlding practices"] se convirtieron una en parte de la otra para componer la conexión parcial que caracteriza la vida de los residentes indígenas y no indígenas de muchas regiones andinas » (ibid.). En los documentos los « seres-tierra » se convierten en geografía (montañas, ríos, lagos, lagunas, rocas, etc.) y la tierra en una preocupación legal tanto para indígenas como para hacendados; pero, al mismo tiempo y como telón de fondo, está presente la indisolubilidad entre runakuna y tirakuna y la tierra es también una cuestión de posesión ancestral, no sólo de propiedad. Todo esto plantea el enigma de que fueron « actores y prácticas ahistóricas los que hicieron posible un objeto histórico, [un] archivo, y un proceso histórico trascendental, la reforma agraria » (p. 149). He aquí precisamente donde reside la complejidad ontológica del archivo de Mariano, y « esta complejidad sugiere que mientras los runakuna comparten nuestra historia, sus vidas también la rebasan » (p. 151).

6 Las décadas transcurridas entre la ejecución de la reforma agraria y el comienzo de este siglo fueron testigo de la expansión de un mercado - en un contexto de decaimiento de la producción agrícola regional - en el que el «multiculturalismo neoliberal fue un instrumento y una consecuencia » (p. 163). A este proceso, en el cual el mercado está cada vez más interesado que el Estado en el reconocimiento de la diferencia, se asocia la expansión de la industria del turismo místico global y, en los Andes, la proliferación del chamanismo andino - auspiciada por distintos sectores de la sociedad regional, nacional e internacional tales como agencias de turismo, antropólogos, políticos, etc. - en la que el hijo de Mariano Turpo fue uno de sus exponentes. En este contexto, parte de la vida de Nazario ejemplifica el viraje, sin anularla necesariamente, de la condición de yachaq a la de chamán ${ }^{4}$, convirtiéndose así también en un exponente de una "religión indígena » que no lo es tal para aquellos que la encarnan. Es decir, la relación entre tirakuna y runakuna es y no es, al mismo tiempo, religión. Lo que la transformó en religión fue un largo proceso de conversión. Ahora bien, « la conversión implica traducción, y explorar la traducción puede revelar la complejidad ontológica de estas entidades [...] y su participación en formaciones culturales diferentes, y parcialmente conectadas, donde ellas son más que uno y menos que muchos» (p. 205-206). Así, en tanto "religión indígena ", resultado de un largo proceso de conversión que encuentra sus fundamentos en formaciones culturales que profesan la división naturaleza-cultura, los tirakuna son naturaleza o, en todo caso, demonios, pero en tanto no religión, « son seres que están con los runakuna en colectividades socio-naturales que no respetan la división entre Dios, naturaleza y humanidad» (p. 206). Nazario Turpo fue también un exponente de todo esto, y quizás por ello mismo el capítulo relativo a su participación como colaborador de la exhibición de la "comunidad quechua» en el National Museum of American Indian inaugurada en 2004 adopta ribetes de comedia (el capítulo efectivamente se titula « Una comedia de equívocos»), entre los que destacan las "fricciones de representación" ( ( frictions of representation», p. 218) entre Nazario y su contraparte del museo, Emil Her Many Horses, un artista ex jesuita de la nación sioux Oglala Lakota (p. 216).

7 El último capítulo del libro discute la « misión biopolítica » («biopolitical mission », p. 249) del Estado moderno - « dejar morir a los runakuna para hacerlos vivir como 
ciudadanos modernos » (ibid.) -, así como el rol que desempeñan las rondas campesinas cuya organización descansa en la "relacionalidad del ser en-ayllu» («in-ayllu relationality ", p. 258) - en el quehacer político regional y nacional en donde se revela un escenario en el que « regímenes no-representacionales [el de las rondas] cohabitan con modos de representación que son la norma del Estado moderno » (p. 269). Finalmente, y en la misma dirección, en el epílogo se discuten otros fenómenos contemporáneos que atentan directamente contra la existencia misma del ayllu tales como la expansión de la industria minera. En esta arena de conflicto la autora advierte la emergencia de momentos de « desacuerdo ontológico » (« ontological disagreement », p. 279) que, lejos de ser « cosmopolitas », son más bien « cosmopolíticos» (« cosmopolitical moments", ibid.) y, como tales, plausibles de proponer una « política-otra » (« alter-politics », ibid.) a la « política moderna » (« modern politics », p. 283), una propuesta que se ajusta a la del libro: « una propuesta etnográfica cosmopolítica » (« an ethnographic cosmopolitical proposal », p. 280).

8 Aunque no es posible en el espacio de una reseña discutir todos los problemas desplegados a lo largo del libro, no quisiéramos dejar pasar por alto, aunque más no sea brevemente, la manera en que se ha conceptualizado el " ayllu». Al decir de la autora, " ayllu es un término ubicuo en el registro etnográfico andinista, usualmente definido como un grupo de humanos y personas otras-que-humanas ("persons other-thanhuman») relacionadas entre sí mediante vínculos de parentesco y que habitan colectivamente un territorio que también poseen» (p.43). Aunque admite estar familiarizada con este tipo de definiciones, la autora confiesa que una conversación con Justo Oxa - un maestro quechua hablante de escuela primaria - le permitió hacer un uso más amplio del término "ayllu» como concepto etnográfico, que revela una «modalidad de relación» (« relational mode», p. 44) que no encontró en sus lecturas andinistas: « el ayllu es un colectivo de humanos, seres otros-que-humanos (" otherthan-human-beings»), animales y plantas conectados intrínsecamente de tal modo que nadie dentro de él escapa a la relación, al menos que ella (él o eso) quiera desafiar el colectivo y arriesgue separarse de él» (p.44)5. Eso no es todo: «el lugar es el acontecimiento de la relacionalidad en-ayllu («in-ayllu relationality») del cual emergen también los runakuna y los tirakuna - no hay separación entre runakuna y tirakuna, ni entre ellos y el lugar. Ellos son todos en-ayllu, la relación de la cual ellos emergen siendo (« being ", p. 101 - ver también p. 133). Esta conceptualización es la que permite a la autora proponer un concepto de ayllu alternativo al que utilizan de manera frecuente, según sus propias palabras, los políticos de izquierda y los antropólogos: « el primer grupo está interesado en la propiedad colectiva de la tierra que el ayllu supuestamente manifiesta; el segundo tiende a hacer foco en las relaciones de parentesco entre los humanos que habitan esa tierra. Ambas nociones siguen las distinciones habituales entre humanidad y naturaleza, y definen el ayllu como un grupo de humanos que habitan un territorio y que están relacionados a través de relaciones que pueden ser económicas o rituales. Esto no está mal, por supuesto » (p. 101). Finalmente, el Estado desconoce o no reconoce la existencia de ayllus en el interior del territorio nacional y " comunidad campesina " es " el nombre que el Estado usa para algunas aldeas rurales » (p. 11) o, en otros términos, es « la designación oficial » de los ayllus (p. 156).

9 La caracterización precedente no deja de presentar problemas. En primer lugar, entre la primera definición de ayllu con la que la autora dice encontrarse familiarizada y aquella a la que arriba luego de reflexionar sobre la que le fue proporcionada por Justo Oxa, no encontramos una diferencia cualitativamente significativa que vaya más allá de los giros 
filosóficos de la segunda: en las tres hay seres humanos, otras cosas y sitios (trátese de territorios, lugares o tejidos), relacionados entre sí sin mayor distinción entre naturaleza y hombres (i.e., hombres/mujeres/cosas, mujeres/hombres/cosas, cosas/mujeres/ hombres, etc.). Ciertamente, en la primera definición los humanos y las otras cosas están relacionados mediante vínculos de parentesco - a semejanza de los hilos en un tejido ${ }^{6}$-; en la segunda definición, en cambio, ello no es necesaria ni prioritariamente así. En segundo lugar, no son sólo los antropólogos (no nos interesa pronunciarnos sobre lo que opinan los políticos de izquierda, ni tampoco sobre si, en efecto, «los» antropólogos han opinado sobre la materia en los últimos quince años) quienes consideran que el ayllu es un grupo de personas relacionadas entre sí mediante vínculos de parentesco y que habitan un territorio determinado: son los mismos campesinos quechua hablantes de la misma zona en la que trabajó la autora quienes opinan lo mismo y que incluso se han pronunciado sobre el particular con precisión meridiana (Sendón 2006, p. 30-31 y 40). En tercer lugar, la « comunidad campesina » en el Perú contemporáneo no es el « nombre » con el que el Estado designa a algunas aldeas rurales; es una institución - metamorfosis mediante - que regula las formas de ocupación y usufructo de aquellas porciones del territorio nacional cedidas a las poblaciones campesinas, indígenas y nativas que no sólo se identifican sino que también son bien conscientes de los mecanismos que definen e involucran su pertenencia a ellas. Las comunidades, por otra parte, también tienen sus respectivos nombres. Además, la «comunidad» y el «ayllu» son dos cosas bien distintas, y si bien pueden coexistir en el interior de una misma población, ello no oblitera el hecho de su distinción por parte de las mismas poblaciones organizadas en una y otro ya que son ellas las que practican cotidianamente ambos modos de relación con los hombres, la naturaleza y demás. En el libro, sin embargo, al menos en dos oportunidades se confunden ambas categorías: por un lado, la autora cuenta que « el ayllu eligió a Mariano para caminar la queja debido a su habilidad para negociar con el hacendado y con los seres tierra. La comunidad había consultado a las hojas de coca y al Taytacha (Jesucristo) y ambos habían aprobado la elección » (p. 46, énfasis agregado); por el otro, es Justo Oxa quien afirma que « la comunidad, el ayllu, no sólo es un territorio donde vive un grupo de gente, es más que eso » (p. 102, énfasis agregado).

Estos problemas, a su vez, ameritan algunas precisiones. En primer lugar, en la actualidad la totalidad de las poblaciones campesino-indígenas que habitan el macizo del Ausangate están organizadas en comunidades campesinas como resultado de un proceso de metamorfosis jurídico-institucional que comienza en la década de 1920. Se sabe, con mayor o menor precisión, que algunas de estas poblaciones presentan un tipo de organización que remite a una forma de organización en ayllu a propósito de su toponimia, su paisaje, su morfología, sus rituales, su mitología y, entre otras cosas también, su propia historia. En efecto, la presencia de ayllus en la región, cuando han sido debidamente identificados, es fácil de rastrear hasta fines del siglo xVIII y, con algo de mayor dificultad, hasta algún momento después del segundo cuarto del siglo XVI. Mientras que el Estado reconoce oficialmente la existencia de comunidades campesinas en el interior del territorio nacional, no reconoce, o simplemente desconoce - o más precisamente, dejó de reconocer desde hace aproximadamente cinco o seis décadas -, la existencia de ayllus. Esto no significa que el Estado, debido a su propia ceguera ontológica, desconozca la indisolubilidad de contrarios ajenos a su propio ejercicio de clasificación, sino sólo que dejó de reconocer por motivos que ahora no vienen al caso unidades administrativas (i.e., los ayllus) que formaban parte también de su realidad social, política y legal hasta bien entrado el siglo xx, tal y como lo dejan advertir 
diversos tipos de fuentes disponibles en los archivos locales (libros de matrículas, censos de población, expedientes legales, libros parroquiales, actas de comunidad y un gran etcétera). En segundo lugar, el ayllu no sólo ha sido una unidad administrativa reconocida por el Estado (republicano y virreinal), sino que también ha sido, y sigue siendo, una unidad social de contornos más o menos precisos cuyos fundamentos descansan en las efectivas relaciones de parentesco que involucran a sus miembros y que los someten a un ejercicio de clasificación pautado por el lenguaje del parentesco. Estos fundamentos - tampoco reconocidos por el Estado y hacia los cuales incluso atentó el diseño de las políticas de comunidad en cualquiera de sus manifestaciones -, que no son sino el sustrato material del ayllu, permiten identificar la plástica que le es propia, su dinámica interna y las transformaciones al que está sometido con el traspaso del tiempo. En efecto, de relajarse las relaciones de parentesco que unen e identifican a los miembros del ayllu hasta el punto de hacerse inexistentes, el ayllu mismo deja de existir, y viceversa, mediante el establecimiento de las efectivas relaciones de parentesco que unen e identifican a sus miembros, el ayllu cobra vida; entre estas dos opciones se abre un amplio catálogo de posibilidades ${ }^{7}$. Así visto, el ayllu no es un conjunto de actores y prácticas ahistóricas que promueven acontecimientos históricos; el ayllu es una realidad histórica compuesta de seres históricos que experimentan y ejecutan acontecimientos históricos del que son testigos, como hemos mencionado, buena parte de la toponimia, el paisaje, la morfología, los rituales, la mitología y la historia de muchos de las aldeas, los pueblos, las comunidades y también los ayllus que habitan y habitaron el macizo del Ausangate ${ }^{8}$. Dicho esto, y en tercer lugar, es justo observar que si bien se han ensayado desde el siglo XVI varias definiciones del término « ayllu », para la gran mayoría del tipo con la que la autora admite estar familiarizada, no abundan descripciones minuciosas y pormenorizadas que se concentren en los pormenores de su arquitectura interna. Consecuentemente, se sabe bastante poco del ayllu en tanto forma de organización social o, en los términos planteados más arriba, del sustrato material del ayllu. Para ponerlo en términos positivos, son raros - siendo benévolos - los estudios antropológicos dedicados al ayllu que se edifiquen sobre sólidas bases de datos relativos a los efectivos vínculos de parentesco existentes entre sus miembros constitutivos como predican las respectivas definiciones ensayadas en ellos - y si alguno lo hace lo desconocemos. Ahora bien, toda propuesta de una definición alternativa de ayllu que dé " por supuesto » (p. 101) lo que otras definiciones, en este caso " antropológicas ", han propuesto sin haberlo en rigor demostrado para de inmediato abocarse a cuestiones de su propia incumbencia ni siquiera contempladas en ellas, corre el riesgo de transformar el ayllu en una caricatura ${ }^{9}$ y, en el ínterin, el guiño de proponer diálogos también alternativos con las antropologías de otras latitudes e incluso otras disciplinas ajenas corre el riesgo de convertirse en mueca.

11 Un último cometario. El libro está repleto de anécdotas y observaciones autoreferenciales. La última de ellas confiesa que escribirlo le demandó a la autora « un largo tiempo » (p. 290). Leyéndolo, lo mismo experimentó el autor de estas líneas. 


\section{BIBLIOGRAFÍA}

\section{LEGOAS PENA Jorge}

2015, Ontographie du rapport à la terre à Chillimocco: Rationalité et ritual dans l'agriculture d'un village andin au Pérou, tesis doctoral, Université Laval, Québec.

ossio Juan M.

1981, «Expresiones simbólicas y sociales de los ayllus andinos: el caso de los ayllus de la comunidad de Cabana y del antiguo repartimiento de los Rucanas-Antamarcas ", in Amalia Castelli, Marcia Koth de Paredes y Mariana Mould de Pease (eds.), Etnohistoria y antropología andina: segunda jornada del Museo Nacional de Historia, Museo Nacional de Historia/Comisión para el Intercambio Educativo entre los Estados Unidos y el Perú, Lima, p. 189-214.

\section{RICARD LANATA Xavier}

2007, Ladrones de sombra. El universo religioso de los pastores del Ausangate, Instituto Francés de Estudios Andinos/Centro de Estudios Regionales Andinos Bartolomé de las Casas, Lima.

SENDÓN Pablo F.

2006, «Los términos de parentesco quechua qatay y qhachun según los registros etnohistóricos y etnográficos: una interpretación ", Revista Andina, 43, p. 9-58.

\section{NOTAS}

1. A decir verdad, la problemática en cuestión parece estar acaparando la atención de antropólogos dedicados a los Andes. Al respecto, véase por ejemplo la tesis doctoral de Jorge Legoas Pena (2015) que, desde su propio título, anuncia que el término « etnografía » le queda chico y es reemplazado, neologismo mediante, por el término « ontographie ».

2. En el índice del libro el nombre de la provincia es consignado sin « $\mathrm{s}$ » final. Sin embargo, en las únicas dos ocasiones donde ha sido registrado en el cuerpo del texto fue consignado como Quispicanchis - con «s » final (p. 271-272).

3. O chamán, en el sentido preciso que adopta este término en la región, íntimamente asociado a lo que en este libro se llama « seres-tierra " y en otro estudio consagrado particularmente a la materia se denomina "mundo-otro » (Ricard Lanata 2007, p. 54 passim).

4. En el sentido contrario al consignado en la nota anterior.

5. La definición de Justo Oxa es la siguiente: «El ayllu es como un tejido, y todos los seres del mundo - gente, animales, montañas, plantas, etc. - son como hilos, nosotros somos parte del diseño. Los seres en este mundo no están solos: del mismo modo que un hilo en sí no es un tejido, y los tejidos son con hilos, un runa es siempre en-ayllu con otros seres, eso es ayllu » (p. 44).

6. Por ejemplo: «Más allá de un correlato estadístico preciso, los kuraq, en términos estructurales, son aquellos puntos en el tejido parental que hacen efectiva la perpetuidad de una determinada línea mediante el establecimiento de alianzas matrimoniales con las mujeres de otras líneas » (Sendón 2006, p. 32). 
7. Entre estas posibilidades debe ponderarse también la de « ayllu no localizado » que se ha identificado no para la región del Ausangate sino para la sierra central del Perú. Por « ayllu no localizado » se entiende « una unidad social que carece de base territorial, que tiene fronteras definidas, que opera como grupo de trabajo y cuya naturaleza se define más por consideraciones de carácter simbólico que por consideraciones que se refieren al parentesco » (Ossio 1981, p. 192).

8. Aunque es tema de otra discusión, no puede pasarse por alto que, si bien desde la invasión y conquista europea el ayllu ha coexistido con otras formas de sociabilidad, en el último siglo parece haber cedido sitio $\mathrm{y}$, en no pocos casos, haber sido desplazados por ellas.

9. Puede parecer una nimiedad, pero el lector interesado en el número de personas que residen hoy en Pacchanta deberá buscar dicha información en otro libro.

\section{AUTORES}

\section{PABLO F. SENDÓN}

Conicet, Argentina 